\title{
ROSAI DORFMAN DISEASE MANAGEMENT, ACTIVE INTERVENTION OR WAIT AND WATCH: A CASE SERIES
}

\author{
Authors: Manoj Sharma* (1) N. Vishnu S. Reddy (2) Rajesh Gudipudi (3) Priyanka Sharma (4) Rushil Saket \\ Narapareddy (5) \\ Authors Affiliation: (1) Consultant E.N.T Specialist, Community Health Centre, Nakaha, Lakhimpur Kheri U.P. \\ (2) Clinical Director, Head of the Department,(3) Consultant ENT surgeon, Department of Otolaryngology, \\ Care hospitals, Banjara Hills, Hyderabad, Telangana, (4) Consultant ENT Surgeon, District Hospital, \\ Lakhimpur Kheri, Uttar Pradesh, (5) Research Assistant, Arizona State University, Phoenix, USA
}

\begin{abstract}
Rosai-Dorfman disease (RDD) is a rare histiocytic disorder commonly manifested as histiocytic proliferation of lymph nodes and extra nodal tissues initially described as a separate entity by Rosai and Dorfman under the term sinus histiocytosis with massive lymphadenopathy (SHML) in 1969. The treatment strategies can be variable and based on the severity of disease, vital organ involvement and possible complications.
\end{abstract}

The first case was a 47 years old female presenting with nasal obstruction and submandibular lymphadenopathy since few days. The second case was a 8 year old girl presented with stridor and lymphadenopathy in the submandibular region.

In both the cases spontaneous resolution was expected and intervention was done only if there werecomplications. Patients were followed up every three months for two years and both the cases have resolved completely without any sequelae of the disease.

Here we present two cases of Rosai-Dorfman disease presented to our hospital, this case series adds to the growing knowledge of Rosai-Dorfman disease, along with understanding of pathophysiology, clinical diagnosis, treatment strategies and recovery options in cases where complications occurred.

\section{INTRODUCTION}

Rosai-Dorfman disease (RDD) also known as sinus histiocytosis with multiple lymphadenopathy (SHML), is a rare histiocytic disorder which involves the over production of a type of white blood cell called non-langerhans sinus histiocyte. RDD is an idiopathic disease, but some evidence suggests that immune dysfunction and viral infections, such as Human Herpes Virus (HHV), Parvo virus B19, and EpsteinBarr virus (EBV) may play a role in the pathogenesis1-5. However, in vitro hybridization studies for EBV encoded RNA have shown the RDD histiocytes to be negative6. Therefore, the definitive identity to an infectious agent behind RDD remains undetermined.

The diagnosis of RDD is from its typical presentation and histopathological confirmation. It is characterized by painless bilateral massive multiple lymphadenopathy with frequent extranodal site involvement. A biopsy of the involved lymph node and from the involved extra-nodal site usually confirms the diagnosis. The true number of RDD cases is not known, although it does occur worldwide and seems to affect an equal number of males and females. It is most commonly seen in the first 10 years of life, but it can also occur in adult patients.

The course of RDD is unpredictable with episodes of exacerbation and remissions that could last for many years. However, the disease is usually self- 
limiting but $5-11 \%$ patients die from the disease. Treatment is necessary only in those cases in which lymph nodes or extra nodal tissue enlargement induces important clinical signs, such as upper airway obstruction or other vital organ compression. When required, the most used therapeutic strategies include surgery, corticosteroids, chemotherapy and in rare cases radiotherapy.

Case 1: A 47 year old female presented with nasal obstruction for one and half years and a sudden onset of painless bilateral cervical lymphadenopathy in submandibular area for a few days. Nasal endoscopy showed a pinkish mass in both nasal cavities. Computed tomography (CT) imaging showed a soft tissue shadow in both the nasal cavities extending into the choana and left maxillary sinus. The patient underwent an ultrasound guided fine needle aspiration of the enlarged right submandibular lymph node. Histology demonstrated abundant histiocytes with emperiolesis on Iymphophagocytosis and features consistent with RDD. Immunohistochemistry demonstrated S100 (immunostain) positivity which confirmed it as this histiocytic disorder.

After tissue diagnosis we tried a course of corticosteroids for two weeks, but it was not effective. The self limiting nature of the disease was explained to the patient and she was followed up every three months.

After six months, patient came with the complaints of blurring of vision and proptosis of the right eye associated with exacerbation of lymphadenopathy as bilateral cervical lymph node enlarged. A repeat CT scan showed a mass involving the right orbital apex, ethmoid sinus as well as the previously noted left maxillary sinus (Fig.1). As the patient was at risk of losing vision, due to its involvement of the orbital apex, the right orbital apex was de-compressed endoscopically. Post operatively the vision improved on right side but the proptosis remained. A further course of corticosteroids was given for two weeks post operatively but there was no response and the lymphadenopathy persisted without any progression. No further deterioration of vision occurred but the proptosis remained. After two years of follow up from the initial presentation, there was remission of the disease spontaneously and the patient completely recovered without any other sequelae (Fig.2). There was regression of lymphadenopathy on both sides and the proptosis of right eye also resolved completely.

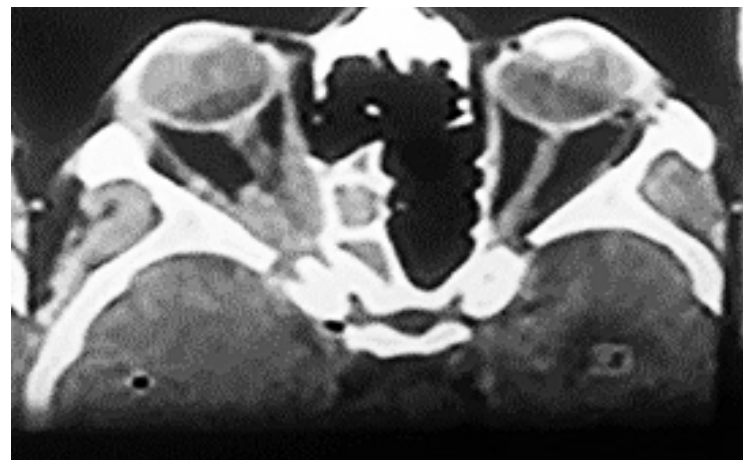

Fig. 1 : CT scan-axial view (Non Contrast): Showing disease involving the right orbital apex.

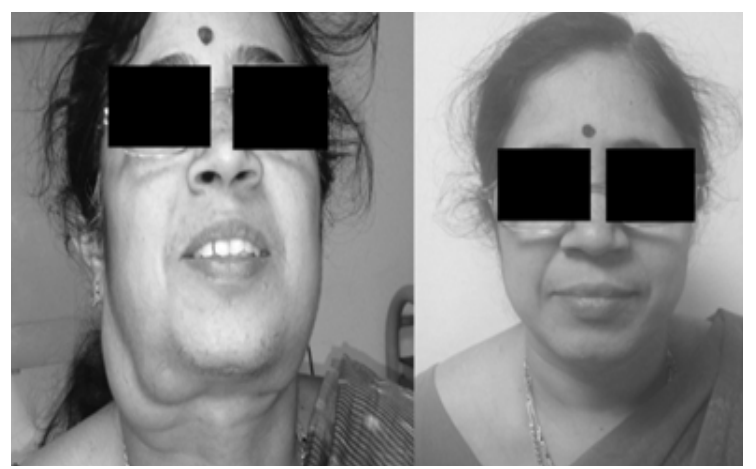

Fig. 2: Image at presentation with multiple lymphadenopathy and after complete resolution

Case 2: The second case was 8 years old girl who presented to our hospital with stridor and bilateral cervical lymphadenopathy. Collateral history revealed that 6 months back she was initially taken to a pediatrics hospital where an excision biopsy of enlarged lymph node at level 1 was done and histopathological examination was done. It was reported as tuberculous lymphadenitis and started on anti-tuberculous therapy but the lymphadenopathy persisted. After six months of treatment she developed mild 
difficulty in breathing initially and within few days presented with stridor in our hospital. A flexible naso-pharyngo-laryngoscopy examination showed a subglottic swelling. As the child was in stridor, an emergency tracheostomy done under local anesthesia followed by micro laryngeal examination and biopsy of the subglottic mass carried out under general anesthesia (Fig.3). Histopathology of subglottic mass was reported as RDD with $\mathrm{S} 100$ and CD 63 positivity with CD1a negativity in immunohistochemisty which confirmed it as the histiocyte disorder, RDD.

The child was treated with intermittent courses of corticosteroids every 4 months for 1 year, but there was no response. Counseling of the patient's parents was done for self limiting nature of disease and wait and watch approach was preferred and child was sent home with tracheostomy tube in situ. Child was reviewed every three months with a lateral soft tissue neck $X$-ray, to monitor for any regression of the subglottic swelling. After following the child for 15 months from the time of tracheotosmy, there was sudden regression of lymphadenopathy and subglottic swelling. The child was decannulatedsuccessfully (Fig.4). On follow up for 12 months after remission in both patients there is no recurrence or sequelae of disease.

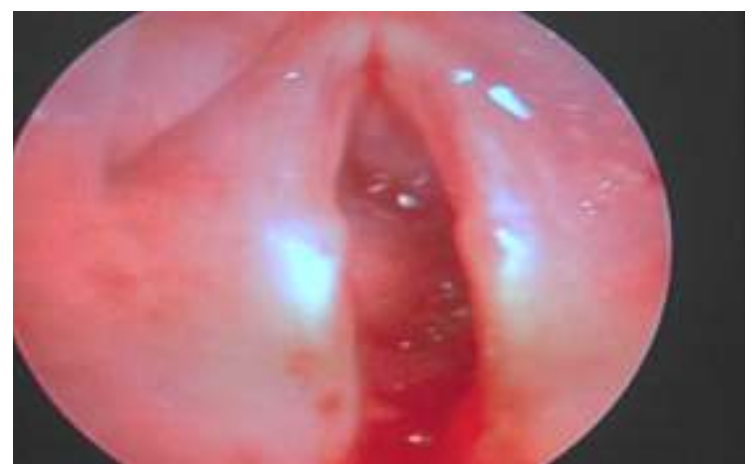

Fig.3 : Intraoperative endoscopic image of larynx showing subglottic obstructive swelling, taken for biopsy

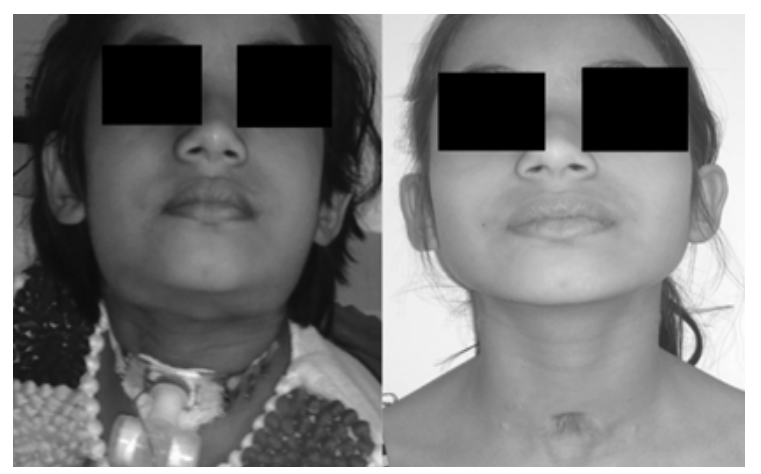

Fig.4 : Image at presentation after emergency tracheostomy and after complete resolution with scar at tracheostomy site after deccanulation.

\section{DISCUSSION}

Rosai-Dorfman disease (RDD) is a rare, idiopathic, histiocytic proliferative disorder. It was first reported by Juan Rosai and Ronald F Dorfman in 19697. The presentation of the disease also varies with the site of involvement. Commonly it presents with painless massive cervical lymphadenopathy but extra-nodal involvement has also been documented in around $40 \%$ of cases, the most common being skin followed by upper respiratory tract and bone. However RDD also can occur in other sites including genitourinary system, lower respiratory tract, oral cavity and soft tissue. Other reported specific sites include brain, spine, liver, kidney, thyroid, breast, parotid gland, orbit, nasal cavity and lungs8. Classically most patients present in otherwise good health with symptoms of fever and massive, painless, cervical lymphadenopathy mimicking lymphoma. Patient may have night sweats and weight loss. Painless maculopapular eruptions also have been reported1.

Emperiopolesis can be found in several haematological diseases, such as myeloproliferative disorders and lymphoma and also in non-malignant disease. This phenomenon consists of the engulfment of cells (commonly lymphocytes) inside another cell (histiocyte). Despite not being a pathognomonic marker for RDD it has been considered an important indicator of this disease and it was a common finding in the pathological lesions of our 
patients9,10,11. An immunohistochemistry analysis is also needed to confirm the diagnosis. In RDD, histiocytes are strongly positive for $\mathrm{S} 100$ protein and negative for CD1a and variably positive for CD6812.

On the basis of literature data and our experience some points can be stressed, despite the variability of treatment approaches reported. If RDD lesions are not massive and do not involve vital organs, the patient should only be observed. In the case of sudden lymph node enlargement with or without fever a corticosteroid course for one to two weeks can be tried to limit the aggravation of disease. If there is any complications like vital organ compression or other life threatening manifestation due to direct nodal or extra-nodal involvement, surgical debulking and or radiotherapy can be performed13. So overall, our experience suggests that following the patient up for a long duration with a wait and watch policy with active intervention whenever needed to treat the complications should be the treatment of choice.

In our two patients we followed similar criteria and had successful outcomes with limited morbidity due to disease or treatment and a complete remission without any sequelae. We are reporting our experience As disease showed spontaneous remission with due course of time, Wait \& Watch should be the treatment of choice unless, any complication develops which requires active intervention. Apart from this we are highlighting in conjunction with literature review that after skin, the respiratory tract is also a common extra-nodal site for RDD which is evident from our two cases involving nose, paranasal sinuses and larynx.

\section{REFERENCES}

1. Samir Dalia, ElizabethSagatys, LumbomirSokol, Timothy Kubal. Rosai-Dorfman Disease: Tumor Biology, Clinical Features, Pathology and Treatment. Cancer Control: Journal of the Moffitt Cancer Centre 2014;21(4):322-7

2. Zhao M, Li C, Zheng J, et al. ExtranodalRosaiDorfman disease involving appendix and mesenteric nodes with a protracted course: report of a rare case lacking relationship to IgG4related disease and review of the literature. Int J ClinExpPathol. 2013;6(11):2569-2577

3. Foucar E, Rosai J, Dorfman R. Sinus histiocytosis with massive lymphadenopathy (Rosai-Dorfman disease): review of the entity. SeminDiagnPathol. 1990;7(1):19-73.

4. Noguchi S, Yatera K, Shimajiri S, et al. IntrathoracicRosai-Dorfman disease with spontaneous remission: a clinical report and a review of the literature. Tohoku J Exp Med. 2012;227(3):231-325.

5. Mehraein $\mathrm{Y}$, Wagner $\mathrm{M}$, Remberger $\mathrm{K}$, et al. Parvovirus B19 detected in Rosai-Dorfman disease in nodal and extranodal manifestations. J ClinPathol. 2006;59(12):1320-1326.

6. Tsang WY, Yip TT, Chan JK. The Rosai-Dorfman disease histiocytes are not infected by EpsteinBarr virus. Histopathology. 1994;25(1):88-90

7. Rosai J, Dorfman RF. Sinus histiocytosis with massive lymphadenopathy. A newly recognized benign clinicopathological entity. Arch Pathol 1969;87(1):63-70

8. Rabindra BhaktaPradhananga, KripaDangol, AnjanShrestha, Dharma KantaBaskota. Sinus Histiocytosis with Massive Lymphadenopathy(Rosai-Dorfman Disease):A Case Report and Literature Review. Int Arch Otorhinolaryngol 2014;18:406-408

9. R.C. Maia, E.de Meis, S. Romano, J.A. Dobbin,C.E. Klumb. Rosai-Dorfmandisease:A report of eight cases in a tertiarty care centre and a review of literature. Brazilian Journal of Medical and Biological Research 2015;48(1):06-12

10. Schmitt A, Jouault H, Guichard J, Wendling F, Drouin A, Cramer EM. Pathologic interaction between megakaryocytes and polymorphonuclear leukocytes in myelofibrosis. Blood 2000; 96(4):1342-1347

11. Chou TC, Tsai KB, Lee CH. Emperipolesis is not pathognomonic for Rosai-Dorfman disease: rhinoscleroma mimicking Rosai-Dorfman disease, a clinical series. J Am AcadDermatol 2013; 69(6):1066-1067

12. Pulsoni A, Anghel G, Falcucci P, Matera R, Pescarmona E, Ribersani $\mathrm{M}$, et al. Treatment of sinus histiocytosis with massive lymphadenopathy (Rosai-Dorfman disease): report of a case and literature review. American Journal of Hematology 2002; 69: 67-71

13. Santra.G, B.K.Das, B.Mandal, S.S.Kundu, A. B a n d o p a d h y a y. R o s a i Dorfmandisease.Singapore Med J 2010;51(10):173-175

\section{*Corresponding Author:}

Dr Manoj Sharma, 692, Avas Vikas Colony Lakhimpur Kheri UP Mobile-+919936868509

Email: dr.sharmamanoj@yahoo.com 